\title{
Article
}

\section{Use of thoracic ultrasound by physiotherapists: a scoping review of the literature}

\author{
Hayward, S.A. and Janssen, Jessica
}

Available at http://clok.uclan.ac.uk/21554/

Hayward, S.A. and Janssen, Jessica ORCID: 0000-0002-5961-2736 (2018) Use of thoracic ultrasound by physiotherapists: a scoping review of the literature. Physiotherapy . ISSN 0031-9406

It is advisable to refer to the publisher's version if you intend to cite from the work. http://dx.doi.org/10.1016/j.physio.2018.01.001

For more information about UCLan's research in this area go to http://www.uclan.ac.uk/researchgroups/ and search for < name of research Group>.

For information about Research generally at UCLan please go to http://www.uclan.ac.uk/research/

All outputs in CLoK are protected by Intellectual Property Rights law, including Copyright law. Copyright, IPR and Moral Rights for the works on this site are retained by the individual authors and/or other copyright owners. Terms and conditions for use of this material are defined in the policies page.

\section{CLoK}

Central Lancashire online Knowledge www.clok.uclan.ac.uk

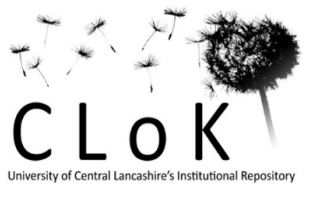


3

ABSTRACT

$4 \quad$ Background

5 Use of diagnostic thoracic ultrasound (TUS) in medical professions to examine the pleura,

7 physiotherapists are using TUS is unclear.

8 Objective

9 The aim of this scoping review is to gain an understanding of the emerging evidence base 10 surrounding physiotherapy use of TUS to inform research and clinical practice.

11 Data Sources

12 A systematic search was conducted of the following databases: Cochrane, EPPI centre, PROSPERO, Medline, CINAHL, AMED, EMBASE, HMIC, and BNI.

14 Study Selection

15 Inclusion criteria: primary research reporting the use of diagnostic TUS; a physiotherapist as 16 part of the study design or as the chief investigator; published in English.

17 Synthesis Methods

Data regarding demographics, design, type of conditions and anatomical structures 19

20 investigated and profession leading the TUS of included papers were compiled in a tabular format. 
22 Of the 26 included papers, 9 studied healthy participants, 4 studied COPD and 4 studied 23 critical care patients. Most papers $(n=23)$ involved scanning the diaphragm. In 8 studies the 24 physiotherapist operated the TUS.

25 Limitations

26 The paper selection process was performed by one author; with no cross-checking by another 27 individual.

28 Conclusion

29 Use of TUS by physiotherapists is an emerging area in both diaphragm and lung diagnostics.

30 A wide range of patient populations may benefit from physiotherapists using TUS. Papers in 31 this review are heterogeneous making any generalisability difficult but does show its potential 32 for varied uses. TUS is an innovative skill in the hands of physiotherapists, but more research 33 is needed.

Funding: National Institute of Health Research (NIHR): Internship programme - no involvement

Contribution of the paper:

- Thoracic ultrasound is gaining popularity amongst physiotherapists.

- The diaphragm is the most investigated structure by physiotherapists. 


\section{Introduction}

A growing body of evidence is now available reporting on the efficacy of thoracic ultrasound (TUS), also known as lung ultrasound (LUS) [1-5]. Within the medical profession TUS has been shown to have improved efficacy in the diagnosis of pulmonary conditions such as pneumonia [1-2] and pleural effusions [3] as well as diaphragmatic dysfunction [4-5] when compared to chest radiography $(\mathrm{CXR})$.

Accurate diagnosis of respiratory conditions is of paramount importance to physiotherapists to enhance treatment selection and monitor treatment effectiveness [6]. TUS may provide an alternative, and more accurate, imaging option for physiotherapists that can be performed at the bedside and in real-time. This bedside imaging technique has been termed point-of-care ultrasound or "POCUS" within the literature. The ability of physiotherapists to perform TUS would allow autonomous imaging and may improve the effectiveness of physiotherapy treatment through more accurate diagnostic ability. Additionally, compared to traditional CXR or computed tomography (CT), TUS does not expose patients to ionising radiation.

A pioneer of POCUS acknowledged that if physiotherapists adopted the use of TUS, existing protocols could change as imaging feedback is instant [7]. Two narrative reviews focused on the potential use of TUS by physiotherapists have previously been published [8-9]. Leech et al reviewed the diagnostic performance of TUS when compared to auscultation and CXR. They found that TUS increased diagnostic accuracy of acute pulmonary pathologies and identify those amenable to physiotherapy treatments. However, they continued to report a lack of specific training standards for physiotherapists to learn TUS [8]. Le Neindre et al focused on the basics of TUS, its semiology and how physiotherapists could apply this in practice. They also highlighted how TUS performed better than CXR and auscultation and should be considered as an outcome measure to inform physiotherapy clinical decision making [9]. Both papers discussed how TUS could help to differentiate between pathologies 
that may or may not respond to physiotherapy treatments thus potentially making physiotherapy interventions more targeted and effective [8-9].

The two previously mentioned reviews did not include a formal search strategy. Neither did they comment on the differing patient populations that may benefit from physiotherapists use of TUS. The aim of this scoping review was to collate the emerging evidence around physiotherapy and the use of TUS in order to create an understanding of how the international physiotherapy community is using TUS to inform their research and clinical practice.

\section{Methods}

This scoping review followed the guidance of Arksey and O'Malley [10] and Levac et al [11]. Its purpose was to examine and present a broad overview of the emerging evidence available irrespective of the quality to identify gaps or common usage, clarify key concepts and report on the types of evidence that address and inform practice in an emerging topic area $[10-11]$.

\section{$\underline{\text { Research Question }}$}

87 The Participants, Concept and Concept (PCC) method [12] has been employed to formulate 88 the following research question: "In what ways do physiotherapists use TUS to inform their clinical or research practice?"

P (Participants) - Human adult and paediatric participants.

C (Concept) - The use of TUS where a physiotherapist was involved. 
C (Context) - Any publication type, except review, was included in this review. No limitation on location, outcome measure or date has been imposed.

\section{$\underline{\text { Objectives }}$}

To explore the current evidence base use of TUS by physiotherapists with regards to:
a) Design
b) Type of conditions investigated
c) Anatomical structures investigated
d) Profession operating the TUS

\section{$\underline{\text { Search strategy }}$}

A systematic electronic search was conducted of the following databases: Cochrane database of systematic reviews, EPPI centre, PROSPERO, Medline, CINAHL, AMED, EMBASE, HMIC, and BNI. The search string was developed to capture as wide a selection of papers as possible. The search was conducted up until November 2016. Following the initial database searches, grey literature searching was performed. A second search using all identified keywords and index terms was then undertaken across all included databases.

"physio*.ti.ab" OR "(physical AND therap*).ti.ab" OR "(respiratory AND therap*).ti.ab" AND "lung*.ti.ab" OR "thora*.ti.ab" OR "diaphragm*.ti.ab" OR "respirat*.ti.ab" OR "chest*.ti.ab" AND

"ultras*.ti.ab" OR "sonogr*.ti.ab". 
118 Papers were included when all the following inclusion criteria were observed:

119 1) primary research reporting the use of TUS.

120 2) involvement of a physiotherapist as part of the study design OR a physiotherapist as the

121 chief investigator (This was achieved by cross referencing with ResearchGate).

122 3) published in the English language.

123 Research abstracts from conference or meeting proceedings were included. There was no

124 limitation of the search based on publication date, or participant age. Papers were excluded

125 when they were review articles or when they involved animal or tissue studies. Hand

126 searching of reference lists were undertaken on the papers deemed eligible to ensure a

127 comprehensive search was undertaken (Figure1).

\section{Data analysis}

Data was extracted and analysed by one reviewer (“X”). Extracted data included: first author, year of publication, country, sample size, study design, subject population, outcome measures, comparison, profession of the ultrasound operator and findings. Methodological design of the papers can be found in the characteristics of included papers table (Table 1).

\section{Key definitions}

"Thoracic ultrasound - TUS"

137 TUS can be used to describe the use of US when examining the pleura, lung parenchyma and diaphragm. LUS can, in some instances, be used to describe the examination of the pleura and lung parenchyma without direct involvement of the diaphragm. These two terms 
along with chest US are used interchangeably throughout the literature. TUS will be the term used throughout this scoping review.

"Physiotherapist"

For ease of consistency throughout this scoping review the term "Physiotherapist" will encompass the roles of a "Respiratory Therapist" or "Physical Therapist". There are international differences between job titles and job roles within physiotherapy and to get a truly global indication of the use of TUS these alternate titles were acknowledged and 147 included.

\section{Results}

A total of 3075 titles and abstracts were identified in the database searches. All titles were evaluated for relevance to the research question. Once shortlisted the remaining papers were screened against the inclusion and exclusion criteria and a total of 3049 papers were excluded. The remaining 26 papers were obtained in full and assessed for their eligibility. An additional 7 papers were identified thorough the hand searching of reference lists. Seven papers were subsequently excluded as physiotherapists were not part of the research design (Figure 1).

The current evidence base of physiotherapy use of TUS.

Following study selection 26 papers were included in this scoping review (Table 1): five randomised controlled trials (RCTs) [13-17], nine cross-sectional studies [18-26], two case series [27-28], four case reports [29-32], five conference abstracts [33-37], one audit [38] (Table 1). Five papers were in conference abstract form only [33-37] without a corresponding full published paper therefore only minimal methodological information could be gathered. Included studies were published over a 19-year period between the years of 1997 and 2016 . No qualitative studies were found that focused on the experiences of physiotherapists using 
165 TUS in their practice or research. Based on the country of the lead author, the majority of 166 studies were conducted by authors residing in Brazil and Australia (Table 1). The 167 participants included in the studies ranged in age from 3 months through to 80 years old.

Of the twenty-six papers nine were performed on healthy subjects $[17,20,22,23,25,28,33-35]$ which accounted for 220 of the total 849 study participants (26\%). Pathologies or conditions investigated included; post-operative upper abdominal surgery $[15,26]$, chronic obstructive pulmonary disease (COPD) [13,14,24,29,30], critical care patients [19,32,36,37], post cerebral vascular accident (CVA) [21], spinal cord injury (SCI) [27,31], morbidly obese [16], adolescents with scoliosis [18] and healthy infants [35] (Table 1).

A clear majority of papers (23 of the 26 ) included in this review involved the use of TUS to scan the diaphragm. The three remaining papers involved scanning the pleura and lung parenchyma $[32,36,38]$. These include a prospective audit on a TUS training curriculum by See et al [38] and two papers on the use of TUS in critical care and included a conference abstract by Riley et al [36] and a case report by Leech et al [32]. See et al [38] is the only paper that looked at the training of physiotherapists in TUS.

Either a radiologist/radiographer (termed sonographer) or one of the research team's physiotherapists would perform the TUS (termed operator). As can be seen in Figure 2, up until 2013 most of the US scanning was performed by a non-physiotherapist. However, since 2013 at least eight of the sixteen papers that did report the profession of the US operator have been physiotherapists. The other six of the remaining eight papers did not report on the TUS operator's profession.

\section{Discussion}

This scoping review explored the current evidence base of physiotherapy use of TUS on study design, type of condition, type of anatomical structures, and professionals operating the TUS. It found that across a widely varying research design, the diaphragm of healthy participants, 
patients with COPD or patients on critical care was most often investigated. In a minority of

191 the papers physiotherapists operated the TUS, and these have taken place since 2013.

192

The evidence of the papers varied widely, ranging from case reports to randomised control trials. Across the included papers there were numerous scanning techniques, used with different methodologies, on a wide range of patients/populations. Very few of them have any overlap making the applicability of TUS in clinical and research practice difficult. The previously mentioned review Leech et al [8] also discussed that it remains unclear how the increased accuracy TUS affords physiotherapists will be applicable without further research.

Healthy participants were used to test reliability or validity of assessing the diaphragm using TUS. This is a normal first step into introducing a new method into a profession [4] and fits with this novel assessment tool. Use of TUS in patients with COPD was reported in five papers and in a critical care environment and four papers (Table 1). It highlights potential areas where the use of TUS by physiotherapists to assess the pleura, lung parenchyma and diaphragm could enhance diagnosis and improve patient outcomes, as previously highlighted by Leech et al [8]. Additionally, this scoping review highlights the potential that TUS might have in other areas such as CVA, morbidly obesity and paediatrics. There are only two papers that have investigated physiotherapists' use of TUS on a paediatric population $[31,35]$. There is a need for significant work into this population especially considering the added safety benefits of US when compared to the ionising radiation of CXR and CT.

A large proportion of the papers looked at the use of TUS to assess the diaphragm as a way to influence physiotherapy practice. The papers included reported on multiple ways to assess diaphragm function. However, those assessments involved numerous different scanning techniques making comparisons difficult. This scoping review has demonstrated a lack of research aimed specifically at the physiotherapy professions use of TUS to assess the pleura or lung parenchyma. 
216 This review found a progressive increase in papers involving physiotherapists use of TUS,

217 with more published in the last three years than in the preceding sixteen, indicating that this assessment tool is gaining in popularity.

Training of physiotherapists in the use of TUS is necessary for the use of this assessment tool to researched further. See et al [38] hinted at the ability of non-medically trained personnel to become proficient in image acquisition and image interpretation. When these skills are used to inform clinical reasoning, it takes the operator beyond the role of a "technician" to that of a professional. This issue is raised by both Leech et al [8] and Le Neindre et al [9] as the greatest challenge facing physiotherapists wishing to gain competency in TUS. This is something that has also been highlighted in the medical literature [1]. Guidance can be found in the international expert statement on training in TUS for non-physiotherapists [39] and much of this information will be relevant to inform future physiotherapy focused training programmes. The difficulty lies in negotiating the medicolegal and governance structures for each individual country depending on that professions scope of practice. In the future it seems prudent to adapt currently existing, robust, competency based US training programmes to meet the needs of physiotherapists as a priority if they are to take advantage of this diagnostic technology.

Many aspects of the use of TUS by physiotherapists warrants further investigation. Robust methods of training for physiotherapists need to be established. Ways in which TUS can be used as a diagnostic tool and as an outcome measure to assess the effectiveness of physiotherapy interventions also warrants further work. There is also a need to show how these new diagnostic and assessment skills, in the hands of physiotherapists, affect patient outcomes and experiences as well as a financial benefit to health providers or society as a whole. 
242 Papers in other languages were excluded from this review which may have added bias to

243 the selection process. As previously mentioned a thorough critical appraisal of the quality of

244 papers within this review was not completed as the aim was to report on as wide a scope of

245 TUS use as possible. The paper selection process was performed by one author; with no

246 cross-checking by another individual leaving this review open to selection bias, however due

247 to the sparsity of papers in this topic the 26 papers included seem a fair representation of the evidence in this area.

\section{Conclusion}

251

Use of TUS by physiotherapists is an emerging area regarding both diaphragm and lung diagnostics. There are a wide range of patient populations that might be able to benefit from physiotherapists using TUS as well as the different applications of TUS itself. The collection of papers in this review is heterogeneous in their research questions, participant populations and methodology. This variety makes any generalisability difficult but does show the potential diverse uses of TUS. The evidence suggests that even within this emerging discipline, critical illness and COPD are two popular areas being investigated. However, robust methods of training for physiotherapists need to be established. The potential of TUS and its impact on patients from diagnosis through to monitoring long term outcomes on society need to be explored. This makes TUS a potentially very novel and innovative skill in the hands of the physiotherapy profession.

Ethical Approval: Ethical approval was not required for this study.

Funding: National Institute of Health Research (NIHR): Internship programme - no involvement 


\section{References}

270

[1] Chavez MA, Shams N, Ellington LE, Naithani N, Gilman RH, Steinhoff MC, Santosham

M, Black RE, Price C, Gross M, Checkley W. Lung ultrasound for the diagnosis of pneumonia in adults: a systematic review and meta-analysis. Respiratory Research. 2014;15:50. DOI:10.1186/1465-9921-15-50

[2] Qian-Jing H, Yong-Chun S, Liu-Qun J, Shu-Jin G, Hong-Yu L, Cia-Shuang P, Ting Y, Fu-

Qiang W. Diagnostic performance of lung ultrasound in the diagnosis of pneumonia: bivariate meta-analysis. International Journal of Clinical and Experimental Medicine. $2014 ; 7(1): 115-121$.

[3] Grimberg A, Shigueoka DC, Atallah AN, Ajzen S, lared W. Diagnostic accuracy of sonography for pleural effusion: systematic review. Sao Paulo Medical Journal 2010;128(2):90-95.

[4] Dubé B-P, Dres M. Diaphragm dysfunction: Diagnostic approaches and management strategies. Journal of Clinical Medicine. 2016;5(113). DOI:10.3390/jcm5120113

[5] Zambon M, Greco M, Bocchino S, Cabrini L, Beccaria PF, Zangrillo A. Assessment of diaphragmatic dysfunction in the critically ill patient with ultrasound: a systematic review. Intensive Care Medicine. 2017;43:29-38. DOI:10.1007/s00134-016-4524-z

[6] Gosselink R, Bott J, Johnson M, Dean E, Nava S, Norrenberg M, Schönhofer B, Stiller K, van de Leur H, Vincent JL. Physiotherapy for adult patients with critical illness: recommendations of the European Respiratory Society and European Society of Intensive

289 Care Medicine Task Force on Physiotherapy for Critically III Patients. Intensive Care Med. 2008;34(7):1188-99. DOI:10.1007/s00134-008-1026-7 
291 [7] Lichtenstein DA. Lung Ultrasound in the Critically III: The BLUE Protocol. Springer

292 International Publishing. 2016:p293 ISBN 978-3-319-15370-4

293

294

295

296

297

298

299

300

301

302

303

304

305

306

307

308

309

310

311

312

313

314

[8] Leech M, Bissett B, Kot M, Ntoumenopoulos G. Lung ultrasound for critical care physiotherapists: A narrative review. Physiotherapy Research International 2015;20:69-76.

[9] Le Neindre A, Mongodi S, Philippart F, Bouhemad B. Thoracic ultrasound: Potential new tool for physiotherapists in respiratory management. A narrative review. Journal of Critical Care. 2016;31:101-109.

[10] Arksey H, O’Malley L. Scoping studies: towards a methodological framework. International Journal of Social Research Methodology: Theory \& Practice. 2005;8(1):19-32.

[11] Levac D, Colquhoun H, O’Brien KK. Scoping studies: advancing the methodology. Implementation Science. 2010;5(1):1-9. DOI:10.1186/1748-5908-5-69

[12] Peters MDJ, Godfrey CM, Mclnerney P. Methodology for JBI scoping reviews. The Joanna Briggs Institute Reviewers' Manual 2015. Adelaide, South Australia: The Joanna Briggs Institute; 2015.

[13] Yamaguti WPS, Claudino RC, Neto AP, Chammas MC, Gomes AC, Salge JM, Moriya HT, Cukier A, Carvalho CRF. Diaphragmatic breathing training program improves abdominal motion during natural breathing patients with chronic obstructive pulmonary disease: $\mathrm{A}$ randomized controlled trial. Archives of Physical Medicine and Rehabilitation. 2012;93:571577. DOI:10.1016/j.apmr.2011.11.026

[14] Rocha T, Souza H, Brandao DC, Rattes C, Ribeiro L, Campos SL, Aliverti A, Dornelas de Andrade A. The manual diaphragm release technique improves diaphragmatic mobility, inspiratory capacity and exercise capacity in people with chronic obstructive pulmonary disease: a randomised trial. Journal of Physiotherapy. 2015;61:182-189.

DOI:10.1016/j.phys.2015.08.009 

breathing exercises, volume and flow incentive spirometry, on diaphragm excursion and pulmonary function in patients undergoing laparoscopic surgery: a randomised controlled trial. Minimally Invasive Surgery. 2016 DOI:10.1155/2016/1967532

[16] Tenório LHS, Santos AC, Neto JBC, Amaral FJ, Passos VMM, Lima AMJ, Brasileiro-

Santos M. The influence of inspiratory muscle training on diaphragmatic mobility, pulmonary function and maximum respiratory pressures in morbidly obese individuals: a pilot study. Disability and Rehabilitation. 2013;35(22). DOI:10.3109/09638288.2013.769635

[17] Souza H, Rocha T, Pessoa M, Rattes C, Brandao D, Fregonezi G, Campos S, Aliverti A, Dornelas A. Effects of inspiratory muscle training in elderly women on respiratory muscle strength, diaphragm thickness and mobility. Journal of Gerentology Series A, Biological Sciences and Medical Sciences. 2014;69(12):1545-1553. DOI:10.1093/Gerona/glu182

[18] Noh DK, Koh JH, You JSH. Inter- and intratester reliability values of ultrasound imaging measurements of diaphragm movement in the thoracic and thoracolumbar curves in adolescent idiopathic scoliosis. Physiotherapy Theory and Practice. 2016;32(2):139-143. DOI:10.3109/09593985.2015.1091871

[19] Baldwin CE, Bersten AD. Alterations in respiratory and limb muscle strength and size in patients with sepsis who mechanically ventilated. Physical Therapy. 2014;94(1):68-82. Jacomino MEML, Paulin E. Assessment of the reproducibility of the indirect ultrasound method of measuring diaphragm mobility. Clinical Physiology and Functional Imaging. 2014;34:18-25. DOI:10.1111/cpf.12058 
340 Journal of Medical Sciences. 2014;7(3): 452-455. DOI: 10.3889/MJMS.1857-

$341 \quad 5773.2014 .0424$

342 [22] Baldwin CE, Paratz JD, Bersten AD. Diaphragm and peripheral muscle thickness on ultrasound: Inter-rater reliability and variability of a methodology using non-standard recumbent positions. Respirology. 2011;16:1136-1143. DOI:10.1111/j.14401843.2011.02005.x

[23] Yamaguti WPS, Sakamoto ET, Panazzolo D, da Cunha Peixoto C, Cerri GG, Alnuquerque ALP. Diaphragmatic mobility in healthy subjects during incentive spirometry with a flow-oriented device and with a volume-oriented device. Journal of Brazilian Pneumonology. 2010;36(6):738-745. DOI:10.1590/S1806-37132010000600011 [24] Yamaguti WPS, Paulin E, Salge JM, Chammas MC, Cukier A, Carvalho CRF. Diaphragmatic dysfunction and mortality in patients with COPD. Journal of Brasilian Pneumology 2009;35(12):1174-1181. DOI:10.1590/S1806-37132009001200003 [25] Yamaguti WPS, Paulin E, Shibao S, Kodaira S, Chammas MC, Carvalho CRF. Ultrasound evaluation of diaphragmatic mobility in different postures in healthy subjects. Journal of Brasilian Pneumology 2007;33(4):407-413. DOI:10.1590/S180637132007000400009

[26] Blaney F, Sawyer T. Sonographic measurement of diaphragmatic motion after upper abdominal surgery: A comparison of three breathing manoeuvres. Physiotherapy Theory and Practice. 1997;13:207-215. DOI:10.3109/09593989709036464

[27] Hardy F, Walker J, Sawyer T. Sonographic measurement of diaphragm movement in patients with tetraplegia. Spinal Cord. 2009;47:832-834. DOI:10.1038/sc.2009.45 displacement during tidal breathing manoeuvres - a reliability study. Australian Journal of Physiotherapy. 1999;45:41-43. 
[29] Yamaguti WPS, Paulin E, Shiboa S, Chammas MC, Salge JM, Ribeiro M, Cukier A,

366 Carvalho CRF. Air trapping: The major factor limiting diaphragm mobility in chronic obstructive pulmonary disease patients. Respirology. 2008;13:138-144. DOI:10.1111/j.14401843.2007.01194.x

[30] Paulin E, Yamaguti WPS, Chammas MC, Shiboa S, Stelmach R, Cukier A, Carvalho CRF. Influence of diaphragmatic mobility on exercise tolerance and dyspnoea in patients with COPD. 2007;101:2113-2118. DOI:10.1016/j.rmed.2007.05.024

[31] Kim JS, Oh DW. Real-time ultrasound imaging biofeedback training in diaphragmatic function in nontraumatic cervical spinal cord injury: a single-subject experimental study. European Journal of Physiotherapy. 2016. DOI: 10.1080/21679169.2016.1247909 ultrasound to improve intensive care management of a deteriorating patient and prevent intubation: a case report. Physiotherapy Theory and Practice. 2015;31(5):372-376. DOI:10.3109/09593985.2014.1003629

[33] Sota T, Wada T, Miyagi M, Tamaki A, Osaki M, Hagino H. The relationship of diaphragm thickening ratio and respiratory muscle strength in young healthy adults. WCPT Congress 2015. Physiotherapy;101(Supplement 1 eS1425). DOI:10.1016/j.physio.2015.03.1382

[34] Schewitz J, Roos R, van Aswegen H, Manda S. The effect of two passive head down diaphragm length in healthy adult individuals. WCPT Congress 2015. diaphragm before and after use of prolonged slow expiration technique in healthy infants: A pilot study. American Journal of Respiratory and Critical Care Medicine. 2014;189:A3238. 
[36] Riley ML, Cork G, Osman L, Ntoumenopoulos G. Does the addition of diagnostic lung

389 ultra-sound impact on chest physiotherapy intervention in critically-ill patients? Journal of the 390 European Society of Intensive Care Medicine. 2014;Supplement 1:S203.

391 [37] Parry S, Sarwal A, Cartwright M, Morris P, Denehy L. Reliability of quantitative muscle 392 ultrasound analysis in the critically ill population. Critical Care Medicine.

$393 \quad 2013 ; 41(12)$ Supplement - 447.

394 [38] See KC, Ong V, Wong SH, Leanda R, Santos J, Taculod J, Phua J, Teoh CM. Lung 395 ultrasound training: curriculum implementation and learning trajectory among respiratory 396 therapists. Intensive Care Medicine. 2016;42:63-71. DOI:10.1007/s00134-015-4102-9

397 [39] Cholley BP. International expert statement on training standards for critical care 398 ultrasonography. Intensive Care Medicine. 2011;27:369-373. DOI:10.1007/s00134-011$399 \quad 2246-9$ 
